\title{
Buchbesprechungen
}

\author{
Doris Beneder · Rudolf Agner
}

(C) Der/die Autor(en) 2020

\section{Gerd Rudolf}

Psychodynamisch denken tiefenpsychologisch handeln. Praxis der tiefenpsychologisch fundierten Psychotherapie

Schattauer Verlag, Stuttgart, 2019, 141 Seiten, ISBN 978-3-608-40015-1, EUR 25,80.

In Deutschland ist die ,Tiefenpsychologisch fundierten Psychotherapie" eine eigenständige Therapiemethode. Für sie charakteristisch ist die psychoanalytische Theorie, eine im Vergleich zur Psychoanalyse jedoch aktivere Behandlungstechnik bei kürzerer Dauer und nieder-frequentem Setting. Die Praxis der tiefenpsychologisch fundierten Psychotherapie steht vor der Frage: wie kann es gelingen, sich vom theoretischen Wissen der Psychoanalyse leiten zu lassen, ohne eine Psychoanalyse durchzuführen? Im vorliegenden Buch versucht Gerd Rudolf diese Frage zu beantworten. Gerade an Berufsjahren jüngere Kolleg_innen werden großen Nutzen aus seinem Werk ziehen können. Auf 141 Seiten vermittelt es einen kurzen,

Doris Beneder $\cdot$ Rudolf Agner $(\bowtie)$ psychotherapie forum, Österreichischer Bundesverband für Psychotherapie Löwengasse 3/3/4, 1030 Wien, Österreich oebvp.psychotherapieforum@ psychotherapie.at aber pointierten (und mit weiterführender Literatur gut ausgestatteten) Überblick über die tiefenpsychologische Praxis - von Diagnostik bis Forschungstätigkeit. Dem Umgang mit strukturell geschädigten Patient_innen ist ein eigenes Kapitel gewidmet, schließlich stellt diese Patientengruppe einen Großteil der tiefenpsychologisch behandelten Population dar. Rudolf hat mit der "strukturbezogenen Psychotherapie“ bereits seinen Ansatz zur Behandlung dieser als „nicht-analysierbar“ geltenden Patient_innen dargestellt. Die Schwierigkeiten strukturgeschädigter Patient_innen werden darin als basale Beziehungsstörung betrachtet und nicht als Ausdruck innerer Konflikte, die durch deutende Interventionen aufgelöst werden können. Seine therapeutische Haltung ist folglich gekennzeichnet, durch ein aktives Stützen der brüchigen Persönlichkeitsstruktur. An dieser Stelle unterscheidet sich die tiefenpsychologisch fundierte Psychotherapie deutlich von den „aufdeckenden“ psychodynamischen Verfahren. Ebenso „unorthodox" ist Rudolfs Offenheit für Interventionen, die nicht dem psychodynamischen Repertoire entstammen. Dass die Behandlung jedoch stets von psychoanalytisch-stringenter Theorie geleitet sein muss, vermittelt das Buch anschaulich durch seinen leichtfüßigen Wechsel zwischen Theorie und Praxis. Gerd Rudolfs jahrzehntelange klinische und wissenschaftliche Erfahrung ist spürbar in der Klarheit mit der er kom- plexe Zusammenhänge vermitteln kann, ohne in Fachjargon zu verfallen. Die Psychodynamik macht auf diesem Weg einen Schritt in Richtung "Allgemeiner Psychotherapie" und findet eine auch für Therapeut_innen anderer Methoden gut lesbare Form. Von größtem Wert ist dieses Buch wahrscheinlich für (angehende) Psychoanalytiker_innen, die niederfrequente Behandlungen auf den Schultern der Psychoanalyse durchführen wollen und nicht in deren Schatten.

Rudolf Agner

Wien, Österreich

E-Mail: rudolf@agner.at

\section{Micha Hilgers}

Der authentische Psychotherapeut. Band II Behandlungstechnik in komplexen Therapiephasen

Schattauer Verlag, Stuttgart, 2019, 152 Seiten, ISBN 978-3-608-40016-8, EUR 41,20 (als e-book EUR 29,99).

Bereits ein Jahr nach Publikation des ersten Bandes erscheint der zweite Band mit dem Untertitel Behandlungstechnik in komplexen Therapiephasen, was eine entsprechende Nachfrage des Fachpublikums nahelegt. Unter komplexen Therapiephasen versteht der Autor einerseits Situationen, die eine herausfordernde Übertragungs- Gegenüber- 
tragungsdynamik in sich tragen und andererseits Situationen in denen Dritte (Gutachter, Kollegen*Innen usw.) auf den therapeutischen Prozess einwirken. Der Autor vertritt einen tiefenpsychologisch orientierten Ansatz, wobei er das interaktionelle Geschehen zwischen Klienten ${ }^{*}$ en und Therapeuten ${ }^{*}$ in ins Zentrum der Aufmerksamkeit stellt und dabei eine authentische Haltung auf beiden Seiten einfordert. Professionelle Authentizität besteht darin, sich sowohl auf den therapeutischen Prozess einzulassen als sich auch wieder davon zu distanzieren, um die Gegenübertragung zu reflektieren und sie im therapeutischen Gespräch fruchtbar werden zu lassen. Die (authentische) Haltung, mit der das therapeutische Gespräch geführt wird, trägt schließlich dazu bei, dass sich die Haltung des/ der Patienten*in zu sich selbst verändert, worüber emanzipatorische Entwicklungsprozesse angestoBen werden.

Auf diesem Hintergrund werden in acht Kapiteln unterschiedliche Themenbereiche erörtert:

1. Frohgemut in die Altersarmut - Armut und Reichtum in der psychotherapeutischen Beziehung: Der Autor vertritt die Ansicht, dass Psychotherapeuten*innen mit wohlhabenden, armen oder prominenten Klienten*innen nicht ausreichend sicher und angemessen umgehen können, weil Psychotherapeuten*innen die „infantilisierenden Abhängigkeitsbedingungen“ (ebd. S. 6) während der Ausbildung erst nach vielen Jahren überwinden und darüber einen adäquaten Umgang mit Geld finden. Selbstverständlich macht es immer einen Unterschied wer einem als Klient*in gegenübersitzt, genauso wichtig halte ich es die Ausbildungsbedingungen zu diskutieren - hier aber einen vorschnellen Zusammenhang herzustellen erscheint mir eher als vereinfachende Darstellung, die der Komplexität des Anliegens nicht gerecht wird.

2. Der Bericht zum Antrag des Patienten - oder schreiben Sie doch, was Sie wollen. In diesem Abschnitt beleuchtet der Autor die schwierige Beziehung zwischen Psychotherapeuten*in und bewilligenden oder ablehnenden Gutachter*innen und plädiert für eine respektvolle und wertschätzende Begegnung auf Augenhöhe.
3. Andere Umstände - Psychotherapie und Mutterschaft: Es gibt unterschiedlichste Situationen, wo sich jede/r Psychotherapeut ${ }^{*}$ in in ,anderen Umständen" befindet, z.B. wenn man selbst schwer erkrankt ist oder nahe Angehörige, wenn man in finanzielle Schwierigkeiten gerät u.v.m. Verantwortungsvoll auch in solchen Lebenssituationen für unsere Patienten*innen da zu sein sollten wir gelernt haben, wenn gleich dies immer eine Herausforderung ist. Warum der Autor gerade die Situation der Mutterschaft hervorgehoben hat, geht zwar aus manchen Aussagen hervor (z.B. die rasante Zunahme weiblicher Kandidatinnen und Psychotherapeutinnen), aber es fehlt eine umfassende Analyse der gesellschaftspolitischen Bedingungen von Mutterschaft und Berufstätigkeit.

4. Angst vor Regression? In diesem Kapitel wird die Bedeutung von erwünschter, weil vorübergehend und den therapeutischen Prozess fördernder und maligner, weil dauerhaft die Übernahme von Verantwortung behindernder, Regression angesprochen. Die Wechselseitigkeit des Prozesses betonend, zeigt der Autor auf, welche Möglichkeiten der/die Therapeut*in hat, um maligne Prozesse (z.B. das Verharren in einer Haltung des Versorgt-werdenWollens oder die Fixierung der Bedürfnisbefriedigung in der therapeutischen Beziehung) zumindest nicht zu fördern. Dazu zieht er zahlreiche Praxistipps aus unterschiedlichsten Therapiesituationen heran und hält ein Plädoyer für gruppentherapeutische Settings. Selbst leidenschaftliche Gruppentherapeutin erlaube ich mir anzumerken, dass natürlich das Setting alleine nicht reicht, um Regression zu vermeiden. Wie dem Autor sicher aus eigener Erfahrung bekannt ist, können auch Gruppen regredieren und gemeinsam wehklagen - wenn man diesen Prozess nicht rechtzeitig erkennt und entsprechend interveniert.

5. Komplexe Therapiesituationen I: Gespräche und Kontakte: In diesem Kapitel werden Situationen mit Dritten (das Gespräch mit den Eltern, Vorbehandlung oder Sprechstundenkontakte bei Kollegen*innen usw.) sowie komplexe Gegenübertragungssituationen (Therapieabbrüche, Setting Wechsel von der Einzel - in Paartherapie, in Coaching/ Supervision, die Bedeutung der Anrede in unterschiedlichen Settings...) erörtert. Hier wird für die Praxis Wichtiges angesprochen, wobei die diese Situationen überwiegend aus der Perspektive des/ der Therapeuten ${ }^{*}$ in betrachtet werden. Hier werden zahlreiche Reflexionsanstöße gegeben.

6. Komplexe Therapiesituationen II: Die Unfähigkeit, begrenztes Glück zu ertragen: Im zweiten Kapitel werden Situationen innerhalb der therapeutischen Dyade (über glückliche Momente, Schweigen, Dauersprechen etc.) angesprochen. Aus der Fülle der dargestellten Situationen greife ich die Überlegungen von Hilgers über Glücksmomente in der Therapie heraus, da er über die gemeinhin übliche „Ressourcenorientierung" in der Psychotherapie hinausgeht und den "chairos", den geglückten Moment anspricht. Achtsam und feinfühlig beleuchtet er die unterschiedlichen Auswirkungen dieser Momente im therapeutischen Prozess.

7. Erweiterte Rahmenbedingungen: Psychotherapie im globalen Turbokapitalismus: In diesem Kapitel wird der Einfluss sozial- und gesellschaftspolitischer Faktoren auf Ausbildung und psychische Gesundheit/ Krankheit angerissen und die emanzipatorische Kraft der Psychotherapie betont. Hilgers fordert auf sich für die Lebensverhältnisse unserer Klienten*innen aufrichtig zu interessieren, mit innen darüber ins Gespräch zu kommen. Darüber hinaus fordert er Psychotherapeuten*innen auf sich angesichts antidemokratischer Tendenzen klar zu positionieren und sich auch politisch mit ihrer Fachkompetenz zu äußern.

8. Das Persönliche ist politisch ... der globale Triumph des Irrationalen: In diesem Kapitel geht der Autor auf narzisstisch-totalitäre Entwicklungen Deutschland und dem europäischen Raum, sowie den USA ein. Er setzt sich für eine Ent-Emotionalisierung der Politik und für klare gesellschaftliche Analysen unseres Berufsstandes ein, wobei er einen vermehrten Rückzug der Psychotherapie ins Private der therapeutischen Situation feststellt und diesen kritisiert.

9. Ein unscharfer Tiger - Bemerkungen zum Coverbild: Das Tigerbild von Eric Peters hinterlässt beim Betrachten den Eindruck eines unscharfen, sich bewegenden Tigers. Diese Metapher für den psychotherapeutischen 
Prozess begleitet den Autor durch das Buch: „Der Tiger, das Selbst bleibt stets verschwommen, ist nie zur Gänze erkennbar. (...) Wir leben in einem steten Bemühen, das sein Ziel nie erreicht - in der Unschärfe unserer Relation zu uns selbst und den anderen." (ebd. S.142)

Das ausgesprochen schön aufgemachte und sorgfältig lektorierte Buch lädt zum Nachblättern ein - leicht zu lesen kann es Reflexion anregen - aber nie ersetzen. Der Schreibstil ist so gewählt, dass er für PsychotherapeutInnen aller Orientierungen grundsätzlich verstehbar ist. In großer Offenheit lässt der Autor die Leser*innen an seinem Erfahrungsschatz teilhaben und spricht offen, auch über eigene Fehler und Unzulänglichkeiten. Die vielen Praxistipps haben manchmal etwas rezepthaftes, dem man widerstehen sollte. An vielen Stellen wäre ich mit dem Autor gerne persönlich ins Gespräch gekommen - insbesondere, wenn sich Widerspruch regte oder mir die seinen Interpretationen zugrunde liegende theoretische Position nicht verständlich war. Dies scheint mir auch der größte Gewinn dieses Buches zu sein, dass es zum offenen Gespräch und zur Klärung der eigenen Positionen und Haltungen anregt.

Doris Beneder

Kottingbrunn, Österreich

E-Mail: doris@dorisbeneder.com

Silke Brigitta Gahleitner, Brigitte Schigl (2019)

Psychotherapie als Beziehung und Prozess. Chancen, Risiken, Fehlerquellen. Reihe: Psychotherapeutische Dialoge $\mathbf{~} \mathbf{H g}$. Britten, Uwe)

Vandenhoeck \& Ruprecht. Göttingen. ISBN 978-3-525-40684-7. EUR15,99

Bei „Psychotherapie als Beziehung und Prozess. Chancen, Risiken, Fehlerquellen“ aus der Reihe „Psychotherapeutische Dialoge" handelt es sich um einen Band, der in seiner Vielstimmigkeit und durch die zahlreichen Querverweise der vielseitigen Wissenschaftlerinnen viel eher wie ein bunter Polylog erscheint. Im mäandernden Gesprächsfluss beant- worten die beiden ausgewiesenen Expertinnen, Silke Brigitta Gahleitner und Brigitte Schigl, ausführlich kurze Fragen von Uwe Britten. Dabei pflichten sie einander bei, widersprechen einander, verlieren und finden sich, entdecken Neues und nehmen auf Vertrautes und Altbekanntes Bezug.

Die Zugänge sind zum Teil kontrovers, auch auf Grund der verschiedenen Arbeitsbezüge der beiden Interviewpartnerinnen. Silke Gahleitner, Professorin für Klinische Psychologie und Sozialarbeit an der Alice Salomon Hochschule in Berlin verweist auf langjährige Tätigkeit in der Sozialen Arbeit im Anti-GewaltBereich, Erfahrung als Psychotherapeutin in eigener Praxis und Beschäftigung mit traumatisierten Kindern und Jugendlichen und jungen Erwachsenen. Brigitte Schigl gilt als Fachfrau für geschlechtsspezifische Themen innerhalb der Psychotherapie und baut auf langjährige Erfahrung als Lehrtherapeutin und Supervisorin in der Ausbildung integrativer PsychotherapeutenInnen und Leiterin des Studienganges Psychotherapie und Beratungswissenschaften an der Karl Landsteiner Universität für Gesundheitswissenschaften in Krems. Sie ist Mitautorin der sogenannten RISK Studie in der „Wirkung, Risiken und Nebenwirkungen von Psychotherapie" empirisch beforscht und ein „Beipackzettel für Psychotherapie" entwickelt wurde.

Das Buch beginnt für ein Sachbuch reich an Atmosphäre mit der Beschreibung der Interviewsituation wie des ,jungen Retrievers Pace, der brav zu unseren Füßen liegt“. Dort bleibt er, ruhestiftend, auch beim weiteren Lesen über Gelingen und Misslingen, über Risiken und Nebenwirkungen von Psychotherapie und die besondere Bedeutung der professionellen Beziehungsgestaltung. Zentral ist dabei das Umgehen mit menschlichen Fehlern im therapeutischen Prozess.

Die beiden Interviewten begegnen sich zu Beginn auf einer Metareflexionsebene hinterfragen metatheoretische Konzepte unter anderem Wahrnehmung, Wirklichkeitskonstruktion, Komplexität und deren Reduktion, Machtstrukturen und Intuition. Darunter versteht Gahleitner „tacid knowledge“, „schlummerndes Wissen", und so benutzt sie bevorzugt den erweiterten Begriff der „strukturierten Intuition“, um nicht als „Bauchge- fühl“ missverstanden zu werden. Schigl ergänzt ein von ihr entworfenes hilfreiches Modell für die therapeutische Praxis "Das exzentrische Dreieck", das aus Therapeut*in, Patient*in und einer Reflexionsebene besteht: „Mich spüren, die Patientin spüren und darüber nachdenken."

Im Kapitel „Risiken von Psychotherapie, Forschung und Alltag“ werden diese mit Beispielen präzise ausdifferenziert. Schigl definiert Nebenwirkung als etwas was neben einer erwünschten Wirkung von Psychotherapie auch auftreten kann, etwa eine größere Labilität zu Beginn der Therapie. Auf diese Möglichkeit sollte Patienten*innen vorab hingewiesen werden. Davon deutlich zu unterscheiden seien Therapieschädigungen, also ein Schaden, der ohne Therapie nicht entstanden wäre, etwa bei ethischen Fehlern, sexueller oder narzisstischer Ausbeutung oder gar Missbrauch. Des Weiteren hat Schigl vier Arten von Fehlern extrahiert, die sie in Folge detailliert an Hand von Praxisbeispielen darstellt: die sogenannten Alltagsfehler, die Fehler in schwierigen Situationen, die Kunstfehler und die schwerwiegenden ethischen Fehler. Gahleitner zitiert eine Studie von Michael Lambert (2015) wonach nur rund ein Prozent der Therapeuten*innen achtzig Prozent der schweren Therapiefehler verursachen. Die Funktionalisierung für eigene Bedürfnisse, das Bedürfnis nach gesteigerter Anerkennung durch andere stünde dabei im Vordergrund.

Mit der Kurzformel „Wissen, Können, Haltung" fasst Gahleitner wesentliche Anforderungen an Psychotherapeuten*innen zusammen. Es ginge darum, immer wieder neugierig zu sein. „Verstehen kann nur gemeinsam entstehen". Wesentlich sei der Umgang mit Fehlern, eine durchlässige, offene und dialogische Art und Weise der Auseinandersetzung, sind sich die Interviewpartnerinnen einig. Das bedeutet im Kontakt mit den Patienten*innen verantwortlich umzugehen, sie wenn möglich vorzuwarnen, zu schützen, etwaige Missgeschicke möglichst als Wachstumsfaktor zu nutzen. Selbstzweifel und Reflexion der Beziehung zu den Patienten*innen seien notwendig, insbesondere da Therapie ja zumeist „behind closed doors“ stattfindet. „Wie dürfen uns gar nicht sicher sein. Das 
wäre ein gefährliches Trugbild." fordert Gahleitner. Psychotherapeuten*innen sind dazu aufgerufen, eigenes Handeln und Verhalten kritisch zu hinterfragen, eigene Unzulänglichkeiten zu verorten. Das verantwortungsvolle Umgehen mit Macht und Machtstrukturen sei bei einem so sensiblen Bereich wie der Psychotherapie notwendig.

Im Kapitel Beziehungsarbeit erörtern die Interviewten ausführlich die bahnbrechende Bedeutung derselben, die eindeutig und erwiesen den Erfolg von Therapie mitbestimmt und beschreiben unter anderem Verlässlichkeit als entscheidenden Parameter. Schigl bringt als anschauliche Metapher den Kleinen Prinzen von Saint-Exupéry ein, bei dem Geduld und behutsames Annähern notwendig gewesen sei, um das Vertrauen des Fuchses zu gewinnen. Gahleitner ergänzt, dass „erst auf dem Boden einer stabilen Beziehung zu neuen Ufern aufgebrochen werden kann." Beziehung sei jedoch jeweils unterschiedlich, es gäbe kein Patentrezept, vielmehr müsse sie in jedem Fall sensibel gemeinsam entwickelt werden. Aufmerksamkeit, solides Wissen und Übung würden dabei helfen situationsadäquat und zugewandt zu reagieren.

Im Kapitel Lösung schwieriger Situationen beschreiben Schigl und Gahleitner, dass es zu allererst um das Wahrnehmen und Erkennen ginge, um das gezielte Ansprechen von schwierigen Situationen. Besonders anschaulich sind die persönlichen Berichte, das offene Mitteilen von Erlebtem und Erfahrenem. Leser*innen können teilhaben an dem reichen Erfahrungsschatz der beiden Autorinnen, die nicht nur theoretisch Fehlerquellen benennen, sondern auch individuelle Lernfelder beschreiben. Das macht Mut und schärft die Aufmerksamkeit im Therapieprozess. Fehlerfreundlichkeit sei notwendig zur weiteren persönlichen Entwicklung, eigene vorgelebte Offenheit in therapeutischen Gesprächen sei vorbildhaft wirksam.

Immer wieder thematisieren die beiden Fachfrauen die geforderte Demut. Patienten*innen könnten zwar begleitet und unterstützt, Selbstexploration gefördert werden. Therapeuten*innen mögen sich jedoch von der Hybris befreien, alle Probleme lösen zu können. Extratherapeutische Faktoren und Entwicklungen seien nicht plan- oder gar steuerbar, es ginge darum auf außerhalb der Therapie liegende Einflüsse konstruktiv einzuwirken. Wichtig sei es eigene Grenzen zu erkennen, Supervision und Intervision zu nutzen und im gegebenen Fall weiter $\mathrm{zu}$ verweisen. Ein anderes Setting, ein anderes Gegenüber könnten neue Möglichkeiten schaffen. Übergänge oder Abbrüche seien gut zu begleiten.

Insgesamt handelt es sich bei dem Buch um ein Lesevergnügen, dass das Kunststück zu Tage bringt, kurzweilig und doch tiefschürfend zu sein. Das Werk hält einiges Relevantes für Anfänger*innen bereit und ruft für Profis Wesentliches in Erinnerung.

Mariella Bruckner

Wien, Österreich

E-Mail: mariella.bruckner@gmx.at

\section{Martin Rufer, Christoph Flückinger (2020, Hg.)}

\section{Essentials der Psychotherapie. Praxis und Forschung im Diskurs}

Hogrefe. Bern. ISBN 978-3-456-85923-1. 144 Seiten. EUR 24,95

„Es ist schon alles gesagt worden - nur noch nicht von jedem." Dieses Zitat von Karl Valentin kommt mir bei Kongressen und Tagungen, aber auch beim Lesen von Fachliteratur oft in den Sinn. Nicht aber bei diesem Buch. Hier stellen erfahrene TherapeutInnen verschiedener Therapiemethoden ihre persönlichen „Essentials der Psychotherapie“ dar, und jeweils spezifisch ausgewiesene ExpertInnen der Psychotherapieforschung reflektieren und kommentieren diese. Das Konzept ist einfach, gut und neu und ganz im Gegensatz zu Karl Valentin fragt man sich, warum nicht schon längst jemand auf die Idee gekommen ist, den psychotherapeutischen und den wissenschaftlichen Diskurs der Psychotherapieforschung in dieser Art zu verschränken. Allerdings muss man wohl 40 Jahre „im Geschäft“ und so gut vernetzt sein wie Martin Rufer, um sowohl namhafte ExpertInnen der Psychotherapieszene wie auch der Forschungswelt zur Mitarbeit an diesem Projekt gewinnen zu können.
Martin Rufer und Hans Lieb sind erfahrene systemische Therapeuten und Autoren wichtiger Fachbücher. Annette Kämmerer und Dirk Zimmer sind Vertreter der Verhaltenstherapie und habilitiert im Fach Psychologie. Verena Kast ist Jung'sche Analytikerin und Professorin im Bereich anthropologische Psychologie. Diese fünf prominenten PsychotherapeutInnen verfassen Beiträge zu so grundlegenden Themen wie den generischen Prinzipien, Psychotherapie und Menschenwürde, kulturspezifischen Aspekten von Psychotherapie, Individualität und Verallgemeinerung in der Fallkonzeption und den Aufbau positiven Denkens im Spannungsfeld von Selbstzweifeln und Selbstakzeptanz. In ihren Essays wird in sehr persönlicher Art jenseits methodenspezifischer Sprachspiele über wichtige Aspekte von Psychotherapie nachgedacht, was auch für erfahrene PraktikerInnen mehr Anregungen und weniger Selbstverständliches enthält, als man von so einem schlanken Buch erwarten würde. Die besondere Bedeutung erhält der Band aber dadurch, dass jeder dieser Essays von einem/einer integrativ-orientierten Psychotherapieforscherln kommentiert und unter Einbezug von Forschungsergebnissen zu diesem Thema weiterentwickelt wird. Auf diese Weise wird die Praxisrelevanz von Psychotherapieforschung deutlich und die viel beklagte Kluft zwischen Forschung und Praxis reduziert.

Als Wissenschaftsbeauftragte einer fachspezifischen Ausbildungseinrichtung lese ich seit einem Jahr regelmäßig das Journal of the Society for Psychotherapy Research. Darin habe ich weniger Anregendes gefunden als in den fünf Beiträgen der in diesem Band vertretenen PsychotherapieforscherInnen. Viele der dort publizierten Forschungsarbeiten beziehen sich nicht auf Fragen, die sich Praktikerlnnen stellen, sondern auf solche, die die Forschung mit ihren etablierten Methoden gut beantworten kann. Praxisrelevanz und empirische Untersuchbarkeit schließen einander zwar nicht grundsätzlich aus, finden empirisch aber doch eher selten zusammen. Nehmen wir das Beispiel therapeutischer Responsivität: Hierunter wird die flexible und individuelle Anpassung der therapeutischen Intervention und Beziehungsgestalt an die konkreten Bedürfnisse des/der KlientIn verstanden. Für die Psychotherapie- 
forschung stellt gelungene Responsivität ein Problem dar. Je individueller TherapeutInnen inr Handeln an den/die PatientIn anpassen, desto schwieriger wird der empirische Nachweis, dass ein bestimmtes TherapeutInnenverhalten oder eine bestimmte Interventionsstrategie erfolgsversprechend ist (vgl. Dinger, S36).

Das große Verdienst von Ulrike Dinger, Christoph Flückiger, Günter Schiepek, Ulrike Willutzki und Maria Borcsa in diesem Band ist es, dass sie ihren Überblick über die gesamte Forschungsliteratur nützen, um jene Forschungsergebnisse zu präsentieren, die für die angesprochenen Themen relevant sind. Dabei geht es weniger um Bestätigung oder Widerlegung der von den „PraktikerInnen“ formulierten Thesen, sondern in vielen Fällen um eine Sensibilisierung dafür, welche Fragen empirische Forschung überhaupt beantworten kann. Das Buch sei allen empfohlen, die sich nicht nur für Psychotherapie interessieren, sondern auch für die Frage, welche Art von Forschung Psychotherapie braucht und welche sie nützen kann. Und dass die Beiträge in ihrer Gesamtheit zum Schluss des Buches in einem Dialog zwischen den beiden Herausgebern, dem Praktiker und dem Forscher, auch noch im gesundheitspolitischen Kontext diskutiert und reflektiert werden, gibt der Essenz noch eine ganz besondere Würze.

\section{Elisabeth Wagner}

Wien, Österreich

E-Mail: wagner.elisabeth@gmx.com

Open Access Dieser Artikel wird unter der Creative Commons Namensnennung 4.0 International Lizenz veröffentlicht, welche die Nutzung, Vervielfältigung, Bearbeitung, Verbreitung und Wiedergabe in jeglichem Medium und Format erlaubt, sofern Sie den/die ursprünglichen Autor(en) und die Quelle ordnungsgemäß nennen, einen Link zur Creative Commons Lizenz beifügen und angeben, ob Änderungen vorgenommen wurden.

Die in diesem Artikel enthaltenen Bilder und sonstiges Drittmaterial unterliegen ebenfalls der genannten Creative Commons Lizenz, sofern sich aus der Abbildungslegende nichts anderes ergibt. Sofern das betreffende Material nicht unter der genannten Creative
Commons Lizenz steht und die betreffende Handlung nicht nach gesetzlichen Vorschriften erlaubt ist, ist für die oben aufgeführten Weiterverwendungen des Materials die Einwilligung des jeweiligen Rechteinhabers einzuholen.

Weitere Details zur Lizenz entnehmen Sie bitte der Lizenzinformation auf http://creativecommons.org/licenses/ by/4.0/deed.de.

Hinweis des Verlags Der Verlag bleibt in Hinblick auf geografische Zuordnungen und Gebietsbezeichnungen in veröffentlichten Karten und Institutsadressen neutral. 